\title{
Sums of nine squares
}

\author{
by \\ Meinhard Peters (Münster)
}

In this paper, the singular series $\varrho_{9}(n)$ for the number of representations of $n$ as a sum of 9 squares is computed in two different ways. This gives a formula for the Dirichlet $L$-series $\sum_{m=1, m \text { odd }}^{\infty}\left(\frac{n}{m}\right) m^{-4}$. Comparing with a result of Lomadze gives a strange identity.

Let $r_{k}(n)$ be the number of solutions $\left(x_{1}, \ldots, x_{k}\right) \in \mathbb{Z}^{k}$ of $x_{1}^{2}+\ldots+x_{k}^{2}$ $=n$. For $k=8$ we have Jacobi's formula

$$
r_{8}(n)=16 \sigma_{3}^{*}(n)
$$

where

$$
\sigma_{3}^{*}(n):= \begin{cases}\sigma_{3}(n), & n \text { odd } \\ \sigma_{3}^{\text {even }}(n)-\sigma_{3}^{\text {odd }}(n), & n \text { even }\end{cases}
$$

with

$$
\sigma_{3}^{\text {even }}(n)=\sum_{\substack{d \mid n \\ d \text { even }}} d^{3}, \quad \text { etc. }
$$

For $k=9$ we have the recursion

$$
r_{9}(n)=\sum_{|s| \leq \sqrt{n}} r_{8}\left(n-s^{2}\right)
$$

Combining (2) with (1) we get

$$
r_{9}(n)=16 \sum_{|s| \leq \sqrt{n}} \sigma_{3}^{*}\left(n-s^{2}\right) .
$$

Now it is known (cf. $[\mathrm{C}]$ ) that the genus of $I_{9}=\langle 1, \ldots, 1\rangle$ (sum of 9 squares) consists of 2 classes, the other one being $E_{8} \oplus I_{1}=: F_{9}$. Denote the number of representations of $n$ by $F_{9}$ by $\alpha_{9}(n)$. Then

$$
\alpha_{9}(n)=\sum_{|s| \leq \sqrt{n}} r_{E_{8}}\left(n-s^{2}\right)=240 \sum_{|s| \leq \sqrt{n}} \sigma_{3}\left(\frac{n-s^{2}}{2}\right),
$$

2000 Mathematics Subject Classification: 11E25, 11E45. 
where

$$
\sigma_{3}\left(\frac{n-s^{2}}{2}\right)=0 \quad \text { if } \frac{n-s^{2}}{2} \notin \mathbb{Z} .
$$

The number of automorphisms of the two classes are:

$$
A_{1}:=\# \operatorname{Aut}\left(I_{9}\right)=2^{9}(9 !), \quad A_{2}:=\# \operatorname{Aut}\left(E_{8} \oplus I_{1}\right)=2^{14} \cdot 3^{5} \cdot 5^{2} \cdot 7 \cdot 2 \text {. }
$$

We have $A_{2}=\frac{15}{2} A_{1}$. Thus the mass of $I_{9}$ is

$$
M\left(I_{9}\right)=\frac{1}{A_{1}}+\frac{1}{A_{2}}=\frac{15+2}{15 A_{1}}=\frac{17}{2786918400}
$$

(cf. [C-S, p. 410]). The average value

$$
\varrho_{9}(n):=\frac{r_{9}(n) / A_{1}+\alpha_{9}(n) / A_{2}}{1 / A_{1}+1 / A_{2}}=\frac{15 r_{9}(n)+2 \alpha_{9}(n)}{17}
$$

can be evaluated by the Minkowski-Siegel theorem. For simplicity we only state the result for $n$ odd and squarefree (see [G, p. 166]):

$$
\varrho_{9}(n)=\frac{\pi^{9 / 2}}{\Gamma(9 / 2)} n^{7 / 2} \mathfrak{S},
$$

where

$$
\begin{aligned}
\mathfrak{S} & =\mathfrak{S}_{2} \frac{2^{9}(8 !)}{\left(2^{8}-1\right) 2^{8} \pi^{8}\left|B_{8}\right|} \sum_{\substack{m=1 \\
m \text { odd }}}^{\infty} \frac{\left(\frac{n}{m}\right)}{m^{4}}, \\
\mathfrak{S}_{2} & =1+\frac{\cos \left(\pi \frac{2 n-9}{4}\right)}{2^{7 / 2}}+\frac{\cos \left(\pi \frac{n-9}{4}\right)}{2^{7}}
\end{aligned}
$$

(the factor 2 in $2 n$ is missing in [G, p. 164, line 4 from bottom]). Here $\left|B_{8}\right|=1 / 30$, so we get

$$
\begin{aligned}
\varrho_{9}(n) & =\frac{24576 n^{7 / 2}}{17 \pi^{4}} \mathfrak{S}_{2} L\left(4,\left(\frac{n}{\cdot}\right)\right), \\
\mathfrak{S}_{2} & = \begin{cases}15 / 16 & \text { if } n \equiv 3 \bmod 4, \\
137 / 128 & \text { if } n \equiv 1 \bmod 8, \\
135 / 128 & \text { if } n \equiv 5 \bmod 8 .\end{cases}
\end{aligned}
$$

Let us check this for $n=1$ :

$$
\varrho_{9}(1)=\frac{24576}{17 \pi^{4}} \cdot \frac{137}{128} \sum_{\substack{m=1 \\ m \text { odd }}}^{\infty} \frac{1}{m^{4}},
$$

where the last sum is

$$
\sum_{m=1}^{\infty} \frac{1}{m^{4}}-\frac{1}{2^{4}} \sum_{m=1}^{\infty} \frac{1}{m^{4}}=\frac{15}{16} \zeta(4)=\frac{15}{16} \cdot \frac{\pi^{4}}{90}=\frac{\pi^{4}}{96},
$$


thus $\varrho_{9}(1)=274 / 17$ (as in Siegel [S, I, p. 369]). On the other hand $\alpha_{9}(1)$ $=2, r_{9}(1)=18$, thus $(5)$ gives

$$
\varrho_{9}(1)=\frac{15 \cdot 18+2 \cdot 2}{17}=\frac{274}{17} .
$$

Comparing (5) with (7) we get a formula for the $L$-series $L\left(4,\left(\frac{n}{.}\right)\right)$, namely

$$
\begin{aligned}
& L\left(4,\left(\frac{n}{*}\right)\right) \\
= & \sum_{\substack{m=1 \\
m \text { odd }}}^{\infty} \frac{\left(\frac{n}{m}\right)}{m^{4}}=\frac{17 \pi^{4}}{24576 n^{7 / 2} \mathfrak{S}_{2}} \cdot \frac{15 r_{9}(n)+2 \alpha_{9}(n)}{17} \\
= & \frac{\pi^{4}}{24576 n^{7 / 2} \mathfrak{S}_{2}}\left(15 r_{9}(n)+2 \alpha_{9}(n)\right) \\
= & \frac{\pi^{4}}{24576 n^{7 / 2} \mathfrak{S}_{2}}\left(15 \cdot 16 \sum_{|s| \leq \sqrt{n}} \sigma_{3}^{*}\left(n-s^{2}\right)+2 \cdot 240 \sum_{|s| \leq \sqrt{n}} \sigma_{3}\left(\frac{n-s^{2}}{2}\right)\right) \\
= & \frac{\pi^{4} \cdot 5}{512 n^{7 / 2} \mathfrak{S}_{2}} \sum_{|s| \leq \sqrt{n}}\left(\sigma_{3}^{*}\left(n-s^{2}\right)+2 \sigma_{3}\left(\frac{n-s^{2}}{2}\right)\right),
\end{aligned}
$$

which is an explicit evaluation after insertion of $\mathfrak{S}_{2}$ from (8).

If $n$ is not odd and squarefree the value for $\varrho_{9}(n)$ is explicitly given by Walfisz ([V, p. 195]):

$$
\varrho_{9}(n)=\frac{24576}{17 \pi^{4}} \mathfrak{S}_{2} L\left(4,\left(\frac{n}{*}\right)\right) n^{7 / 2} \tau_{4}(n)
$$

with

$$
\tau_{4}(n)=\prod_{p \mid n} \frac{1}{1-1 / p^{7}} \prod_{\substack{p \mid n \\ 2 \nmid a}}\left(1-\frac{1}{p^{(7 / 2)(a+1)}}\right) \cdot \prod_{\substack{p|n \\ 2| a, a>0}}\left(1+\frac{\left(\frac{P}{p}\right)-\frac{1}{p^{3}}}{1-\frac{\left(\frac{P}{p}\right)}{p^{4}}} \cdot \frac{1}{p^{7 a / 2+4}}\right),
$$

where $p^{a} \| n, P=p^{-a} n$ and

$$
\mathfrak{S}_{2}=\left\{\begin{array}{l}
\frac{135}{127}-\frac{255}{127 \cdot 16} \cdot \frac{1}{2^{7 h}} \quad \text { if } c=2 h+1 \text { or } N \equiv 3 \bmod 4, c=2 h, \\
\frac{135}{127}+\frac{119}{127 \cdot 128} \cdot \frac{1}{2^{7 h}} \quad \text { if } N \equiv 1 \bmod 8, c=2 h \\
\frac{135}{127}-\frac{135}{127 \cdot 128} \cdot \frac{1}{2^{7 h}} \quad \text { if } N \equiv 5 \bmod 8, c=2 h
\end{array}\right.
$$


where $n=2^{c} N$ (formula (4.59) in [V, p. 195] has a sign error in the middle term).

For $n$ odd and squarefree we get $\tau_{4}(n)=1$, so formula (10) coincides with (7). As before we can evaluate $L\left(4,\left(\frac{n}{*}\right)\right)$ by inserting in formula (10).

Recently Lomadze has shown the following formula (see [L, (1.1)] and [I, p. 187]):

$$
r_{9}(n)=\varrho_{9}(n)+\frac{4}{17} \sum_{x_{1}^{2}+x_{2}^{2}+x_{3}^{2}=3 n}\left(\frac{x_{1} x_{2} x_{3}}{3}\right) x_{1} x_{2} x_{3} .
$$

Comparing this with formula (10) gives

$$
\begin{aligned}
\frac{24576 n^{7 / 2} \tau_{4}(n)}{17 \pi^{4}} \mathfrak{S}_{2} L(4, & \left.\left(\frac{n}{*}\right)\right) \\
& =r_{9}(n)-\frac{4}{17} \sum_{x_{1}^{2}+x_{2}^{2}+x_{3}^{2}=3 n}\left(\frac{x_{1} x_{2} x_{3}}{3}\right) x_{1} x_{2} x_{3} .
\end{aligned}
$$

Inserting (3) we get

$$
\begin{aligned}
& L\left(4,\left(\frac{n}{*}\right)\right) \\
= & \frac{17 \pi^{4}}{48 \cdot 512 n^{7 / 2} \tau_{4}(n) \mathfrak{S}_{2}}\left(16 \sum_{|s| \leq \sqrt{n}} \sigma_{3}^{*}\left(n-s^{2}\right)-\frac{4}{17} \sum\left(\frac{x_{1} x_{2} x_{3}}{3}\right) x_{1} x_{2} x_{3}\right) .
\end{aligned}
$$

Comparing with (9) gives

$$
\begin{aligned}
\frac{17}{48 \tau_{4}(n)}\left(16 \sum_{|s| \leq \sqrt{n}} \sigma_{3}^{*}\left(n-s^{2}\right)\right. & \left.-\frac{4}{17} \sum\left(\frac{x_{1} x_{2} x_{3}}{3}\right) x_{1} x_{2} x_{3}\right) \\
= & 5 \sum_{|s| \leq \sqrt{n}}\left(\sigma_{3}^{*}\left(n-s^{2}\right)+2 \sigma_{3}\left(\frac{n-s^{2}}{2}\right)\right) .
\end{aligned}
$$

Restricting to the case of $n$ odd and squarefree, where $\tau_{4}(n)=1$, we get

$$
\frac{2}{3} \sum_{|s| \leq \sqrt{n}} \sigma_{3}^{*}\left(n-s^{2}\right)-\frac{1}{12} \sum\left(\frac{x_{1} x_{2} x_{3}}{3}\right) x_{1} x_{2} x_{3}=10 \sum_{|s| \leq \sqrt{n}} \sigma_{3}\left(\frac{n-s^{2}}{2}\right)
$$

or

$$
\begin{aligned}
\sum_{x_{1}^{2}+x_{2}^{2}+x_{3}^{2}=3 n}\left(\frac{x_{1} x_{2} x_{3}}{3}\right) & x_{1} x_{2} x_{3} \\
& =\sum_{|s| \leq \sqrt{n}}\left(8 \sigma_{3}^{*}\left(n-s^{2}\right)-120 \sigma_{3}\left(\frac{n-s^{2}}{2}\right)\right)
\end{aligned}
$$

for $n$ odd and squarefree. 


\section{References}

[C] Chao Ko, On the positive definite quadratic forms with determinant unity, Acta Arith. 3 (1939), 79-85.

[C-S] J. H. Conway and N. J. A. Sloane, Sphere Packings, Lattices and Groups, 3rd ed., Springer, 1999.

[G] E. Grosswald, Representations of Integers as Sums of Squares, Springer, 1985.

[I] H. Iwaniec, Topics in Classical Automorphic Forms, Grad. Stud. Math. 17, Amer. Math. Soc., 1997.

[L] G. A. Lomadze, On the number of representations of positive integers as the sum of nine squares, Acta Arith. 68 (1994), 245-253 (in Russian).

[S] C. L. Siegel, Gesammelte Abhandlungen I-III, Springer, 1966.

[V] A. Z. Val'fiš [A. Walfisz], Representations of numbers by sums of squares. Asymptotic formulas, Amer. Math. Soc. Transl. (2) 3 (1956), 163-248.

Mathematisches Institut der Universität

Einsteinstr. 62

D-48149 Münster, Germany

E-mail: petersm@math.uni-muenster.de 